\title{
Intensive culture of Litopenaeus vannamei Boone 1931, in a recirculating seawater system
}

\section{Cultivo intensivo de Litopenaeus vannamei Boone 1931, en un sistema de agua de mar recirculada}

\author{
Benjamín Barón-Sevilla* \\ L. Fernando Bückle-Ramírez \\ Mónica Hernández-Rodríguez \\ Laboratorio de Ecofisiología, Departamento de Acuicultura \\ Centro de Investigación Científica y de Educación Superior de Ensenada \\ Km 107 carretera Tijuana-Ensenada \\ Apartado postal 2732 \\ Ensenada, CP 22800, Baja California, México \\ *E-mail: bbaron@cicese.mx
}

Recibido en abril de 2003; aceptado en octubre de 2003

\begin{abstract}
The intensive culture of Litopenaeus vannamei continues to expand and their study in recirculating rearing tanks with limited water volume is scarce. The aim of this research was to cultivate white shrimp postlarvae (PL 13) for five months in a 5.85- $\mathrm{m}^{3}$ rearing unit. Vertical mosquito screen curtains augmented the tank area of the rearing unit from 13.9 (wall surface) to $84.6 \mathrm{~m}^{2}$. The total water volume $\left(11 \mathrm{~m}^{3}\right)$ was recirculated at a rate of $7.2 \mathrm{~m}^{3} \mathrm{~h}^{-1}$. Shrimp postlarvae were fed with fine-graded Camaronina mixed with water for one month and then replaced with Camaronina pellets distributed automatically twice a day. During the trial, water temperature ranged from $23.0^{\circ} \mathrm{C}$ to $25.8^{\circ} \mathrm{C}$, oxygen from 5.3 to $7.5 \mathrm{mg} \mathrm{L}^{-1}$, and salinity from $27.5 \%$ o to $28.4 \%$. At the end of the experiment, water quality was $\mathrm{pH} 7.4, \mathrm{NH}^{3}-\mathrm{N}=0.8 \mathrm{mg} \mathrm{L}^{-1}, \mathrm{NO}_{2}-\mathrm{N}=0.287 \mathrm{mg} \mathrm{L}^{-1}, \mathrm{PO}_{4}=7.9 \mathrm{mg} \mathrm{L}^{-1}$, and total $\mathrm{PO}_{4}=$ $1.71 \mathrm{mg} \mathrm{L}^{-1}$. The final crop for the unit was $56.7 \mathrm{~kg}$, with a shrimp mean mass of $4.001 \mathrm{~g}$, and a maximum and minimum of 8.484 and $0.384 \mathrm{~g}$, respectively. In terms of the total recirculated seawater volume $\left(11 \mathrm{~m}^{3}\right)$, the production was $5.15 \mathrm{~kg} \mathrm{~m}^{-3}$, and in relation to the tank volume, $9.7 \mathrm{~kg} \mathrm{~m}^{-3}$; considering the tank surface without curtains, production was $4.1 \mathrm{~kg}^{-2}$, and with curtains, $0.67 \mathrm{~kg} \mathrm{~m}^{-2}$. The design and installation of the curtains is a viable tank modification to increase the available space in a reduced water volume. Our results indicate that the mean residence time $(1.23 \mathrm{~h})$ of the water recirculation in the rearing unit can sustain high water quality. Another extension of our results is to make use of the design to culture postlarvae as a nursery unit to raise juveniles less than $4 \mathrm{~g}$ for subsequent transfer to culture facilities. However, it is much more important to implicate in the analysis the use of land that in some countries, such as Mexico, constitutes vast regions of useful agricultural land, transformed into culture ponds to increase the national shrimp production. In this sense, shrimp production of the experiment in terms of the base area $\left(7 \mathrm{~m}^{2}\right)$ of the culture tank was $8.1 \mathrm{~kg} \mathrm{~m}^{-2}$.
\end{abstract}

Key words: intensive culture, production, recirculating seawater system, Litopenaeus vannamei.

\section{Resumen}

El cultivo intensivo de Litopenaeus vannamei continúa expandiéndose y los estudios en estanques de cultivo con sistemas de recirculación con volúmenes limitados de agua son muy escasos. El objetivo de esta investigación fue cultivar postlarvas (PL 13) del camarón blanco durante 168 días en un estanque de $5.85 \mathrm{~m}^{3}$. Se utilizaron cortinas verticales de tela de mosquitero para aumentar la superficie de la unidad de cultivo de 13.9 a $84.6 \mathrm{~m}^{2}$. El volumen total de agua $\left(11 \mathrm{~m}^{3}\right)$ fue recirculada a una tasa de $7.2 \mathrm{~m}^{3} \mathrm{~h}^{-1}$. Las postlarvas fueron alimentadas durante un mes con Camaronina molida, mezclada con agua, y remplazada después con Camaronina peletizada que se distribuyó automáticamente en el estanque dos veces al día. Durante el experimento, la temperatura del agua fluctuó entre $23^{\circ} \mathrm{C}$ y $25.8^{\circ} \mathrm{C}$, el oxígeno entre 5.3 y $7.5 \mathrm{mg} \mathrm{L}^{-1}$ y la salinidad entre $27.5 \%$ y $28.4 \%$. Al final del experimento, la calidad del agua fue de $\mathrm{pH} 7.4, \mathrm{NH}_{3}-\mathrm{N}=0.8 \mathrm{mg} \mathrm{L}-1, \mathrm{NO}_{2}-\mathrm{N}=0.287 \mathrm{mg} \mathrm{L}^{-1}, \mathrm{PO}_{4}=7.9 \mathrm{mg} \mathrm{L}^{-1}$ y $\mathrm{PO}_{4}$ total $=$ $1.71 \mathrm{mg} \mathrm{L}^{-1}$. La cosecha final de esta unidad de cultivo fue de $56.7 \mathrm{~kg}$ y una masa media de los camarones de $4.001 \mathrm{~g}$, con un máximo y mínimo de 8.484 y 0.384 g, respectivamente. En términos del volumen total de agua recirculada (11 $\left.\mathrm{m}^{3}\right)$, la producción fue de $5.15 \mathrm{~kg} \mathrm{~m}^{-3} \mathrm{y}$, en relación con el volumen del estanque, de $9.7 \mathrm{~kg} \mathrm{~m}^{-3}$; considerando la superficie de la unidad de cultivo sin cortinas, la producción fue de $4.1 \mathrm{~kg} \mathrm{~m}^{-2}$ y con cortinas, de $0.67 \mathrm{~kg} \mathrm{~m}^{-2}$. El diseño y la instalación de las cortinas es una modificación viable para incrementar el espacio disponible en un volumen reducido de agua para aquellos organismos que pueden desplazarse a lo largo de las cortinas. Nuestros resultados indican que el tiempo (1.23 h) de residencia del agua recirculada en la unidad de cultivo es adecuado para mantener una alta calidad de los parámetros fisicoquímicos. Otra aplicación de nuestros resultados es utilizar el diseño para cultivar postlarvas como una unidad para crecer juveniles de menos de $4 \mathrm{~g}$ y transferirlos después a estanques de cultivo. Sin embargo, es mucho más importante implicar en el análisis la utilización de la 
tierra que, en algunos países como México, constituye vastas regiones de tierra agrícola transformadas en estanques para incrementar la producción de camarón. En este sentido, la producción de camarón en nuestro experimento con base en el área del estanque de cultivo $\left(7 \mathrm{~m}^{2}\right)$ fue de $8.1 \mathrm{~kg} \mathrm{~m}^{-2}$.

Palabras clave: cultivo intenso, producción, sistema de agua de mar recirculada, Litopenaeus vannamei.

\section{Introduction}

Litopenaeus vannamei Boone 1931 is native to the eastern Pacific Ocean, from the Gulf of California to Peru. This species is studied for its shrimp farming potential in the United States and several countries of Asia and Oceania because it grows faster than some native species. In 1994, the total world production of penaeid shrimp by farms was 741,638 metric tons, of which the giant tiger prawn Penaeus monodon Fabricius 1798 comprised $68.2 \%$ and L. vannamei, $14.8 \%$ (Anonymous, 1996). Aquaculture is one of the fastest growing food-producing sectors, providing an acceptable supplement to substitute for wild fish and plants. From 1984 to 1995, the cultured shrimp and prawn group grew at an average annual compounded rate (APR) of 16.8\%, compared with $2.6 \%$ for fisheries capture. In 1995, 96.5\% of shrimp and prawn production was penaeids. From 1990 to 1995, however, the production rate and expansion of this activity decreased as a consequence of environmental degradation and mismanagement (Anonymous, 1997).

Intensive culture causes waste that increases environmental concerns about coastal contamination, and pond and raceway shrimp culture depends on high water volume with variable effluent water quality. Alternatively, closed water systems offer the possibility to control exposure to extreme environmental changes (thus avoiding the loss of investments) and to improve pollution control.

Great efforts have been made to grow and reproduce shrimp in complete recirculation systems (Ogle, 1991; Ricque et al., 1993; Tirado et al., 1993, 1996; Porchas et al., 1995; Davis and Arnold, 1998), or in floating cages installed in estuarine zones (Casillas and Villarreal, 1995a; Paquotte et al., 1998). However, information on L. vannamei culture in recirculating systems is scarce because commercial farm production dominates the state of the art and continues to expand.

The aim of this work was to study L. vannamei growth in a reduced water volume, at an optimum temperature and salinity, and with tank surface area increased above that found in typical tanks. For aquaculture expansion, it is necessary to continue exploring alternative closed system production to improve water reuse, though conventional production will remain until the former reaches economic viability.

\section{Materials and methods}

\section{Culture system unit description and operation}

The culture system comprises a rearing tank $(3.0 \mathrm{~m}$ diameter $\times 1.1 \mathrm{~m}$ deep, $7 \mathrm{~m}^{3}$ ) connected to a recirculated seawater

\section{Introducción}

Litopenaeus vannamei Boone 1931 es una especie endémica del Océano Pacífico oriental, desde el Golfo de California hasta Perú. Debido a su potencial de cultivo, actualmente se encuentra en estudio en los Estados Unidos y en varios países de Asia y Oceanía, ya que presenta un crecimiento más rápido que el de otras especies nativas. En 1994 la producción mundial de granjas de camarones peneidos alcanzó las 741,638 toneladas métricas, de las cuales el langostino tigre gigante, Penaeus monodon Fabricius 1798, representó 68.2\% y L. vannamei 14.8\% (Anónimo, 1996). La acuicultura es uno de los sectores de producción de alimentos con mayor crecimiento y constituye una alternativa aceptable para complementar la producción de peces y plantas silvestres. De 1984 a 1995 el cultivo de camarones y langostinos creció a una tasa compuesta anual media de $16.8 \%$, en comparación con $2.6 \%$ de la captura pesquera. En 1995 los peneidos constituyeron 96.5\% de la producción de camarones y langostinos; sin embargo, de 1990 a 1995 disminuyó la tasa de producción y la expansión de esta actividad se frenó como consecuencia de la degradación ambiental y un mal manejo (Anónimo, 1997).

Los cultivos intensivos generan desechos que acrecientan la preocupación relacionada con la contaminación costera. El cultivo de camarón en estanques y en sistemas abiertos tipo "raceway" depende de un gran volumen de agua que es descargada con diferentes grados de calidad. Por otra parte, los sistemas cerrados permiten controlar los cambios ambientales extremos (evitando así pérdidas de inversión) y mejorar el control de la contaminación.

Se han hecho grandes esfuerzos para cultivar y reproducir camarones en sistemas de recirculación cerrados (Ogle, 1991; Ricque et al., 1993; Tirado et al., 1993, 1996; Porchas et al., 1995; Davis y Arnold, 1998), o en jaulas flotantes instaladas en zonas estuarinas (Casillas y Villarreal, 1995a; Paquotte et al., 1998). Sin embargo, es escasa la información sobre el cultivo de L. vannamei en sistemas de recirculación, ya que la producción de camarones en granjas convencionales sigue predominando y expandiéndose.

El objetivo del presente trabajo fue estudiar el crecimiento de L. vannamei en un volumen de agua reducido, a temperature y salinidad óptimos, y con una mayor área de superficie que la disponible en los tanques típicos. Para el desarrollo de la acuicultura es necesario buscar alternativas para mejorar el reuso del agua en la producción con sistemas cerrados, ya que de otra manera la producción convencional continuará expandiéndose hasta que aquellos sistemas no alcancen una viabilidad económica. 
system that was purified consecutively through a biological filter, a mechanical filter filled with silica sand, a zeolite filter, and an ultraviolet purifying system (fig. 1) (Spotte, 1979; Anonymous, 1986; Huguenin and Colt, 1989; Timmons and Losordo, 1994). Before drainage from the rearing tank enters the biological filter, seawater passes through a mechanical filter $\left(1.5 \mathrm{~m}^{3}\right)$ divided into five chambers and filled with plastic wool to retain waste material, food particles and faeces (Bückle et al., 1990). The total amount of recirculated seawater was $11 \mathrm{~m}^{3}$ and included minor tanks used for other culture purposes. The mean residence time of the fluid of the large culture tank was $1.23 \mathrm{~h}$ (tank water quantity $\mathrm{m}^{3} \mathrm{~h}^{-1} /$ tank volume $\mathrm{m}^{3}$; Anonymous, 1986).

The wall surface and bottom area $\left(13.9 \mathrm{~m}^{2}\right)$ of the $7.0-\mathrm{m}^{3}$ rearing unit was augmented to $84.6 \mathrm{~m}^{2}$ with 31 vertical mosquito screen curtains upheld from the tank border to an acrylic ring held by the central pipe (figs. 2a-c, 3). The water depth

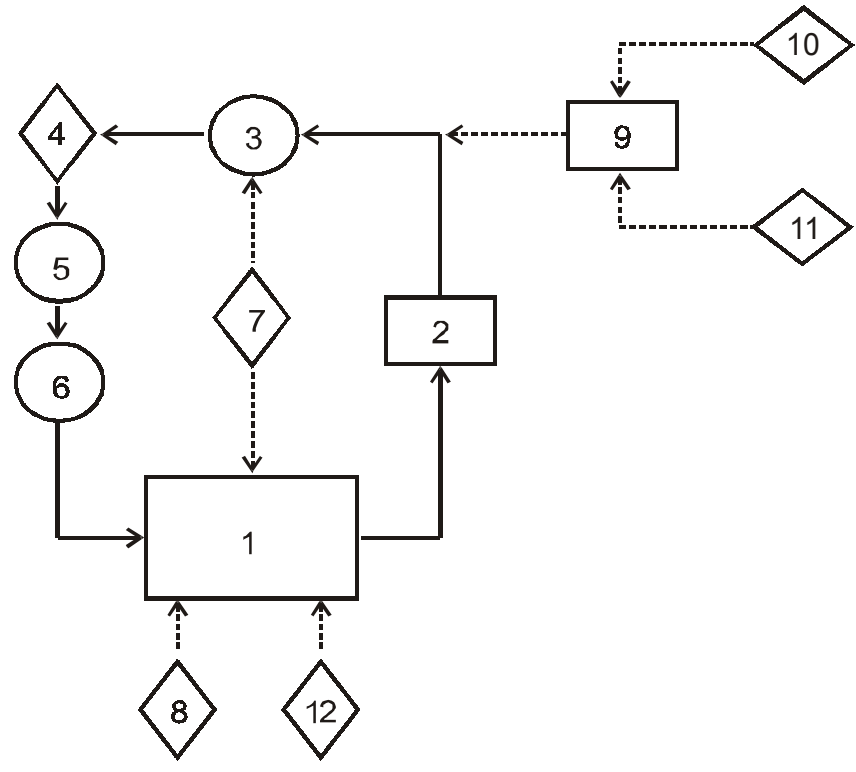

Figure 1. Flow diagram of the whole system: (1) Rearing unit $\left(7 \mathrm{~m}^{3}\right) ;(2)$ waste collecting filter $\left(1.5 \mathrm{~m}^{3}\right)$ divided into five chambers filled with plastic wool; (3) biofilter $\left(3.48 \mathrm{~m}^{3}\right)$ with $3.85-\mathrm{m}^{2}$ surface and $4-\mathrm{cm}$ gravel, ground shell and sand; (4) pump (0.75 HP); (5) high rate sand filter (Triton) with silica sand \#20 (0.046-0.056 mm), maximum flow rate $280 \mathrm{~L} \mathrm{~min}^{-1} ;(6)$ six ultraviolet sterilizers, $40 \mathrm{~W}$ each; (7) air; (8) automatic feeder; (9) seawater reservoir $\left(1 \mathrm{~m}^{3}\right)$; $(10-11)$ fresh or sea water supply to maintain salinity and to compensate for losses due to leaking, evaporation and sand-filter backwash; (12) emergency automatic oxygen system.

Figura 1. Diagrama de flujo de todo el sistema: (1) unidad de cultivo $\left(7 \mathrm{~m}^{3}\right)$; (2) filtro recolector de desechos $\left(1.5 \mathrm{~m}^{3}\right)$ dividido en cinco cámaras rellenas con algodón sintético; (3) biofiltro $\left(3.48 \mathrm{~m}^{3}\right)$ con $3.85 \mathrm{~m}^{2}$ de superficie y 4 $\mathrm{cm}$ de grava, concha molida y arena; (4) motobomba (0.75 HP); (5) filtro (Triton) relleno con arena de sílice \#20 (0.046-0.056 mm) de una capacidad de filtración de $280 \mathrm{~L} \mathrm{~min}^{-1}$; (6) seis esterilizadores con lámparas ultravioleta de $40 \mathrm{~W}$ cada una; (7) aire; (8) alimentador automático; (9) depósito de agua de mar $\left(1 \mathrm{~m}^{3}\right) ;(10-11)$ entrada de agua de mar 0 agua dulce para mantener la salinidad y compensar las pérdidas por evaporación y por la limpieza de los filtros; (12) sistema de oxígeno automático de emergencia.

\section{Materiales y métodos}

\section{Descripción y operación del sistema de cultivo}

El sistema consiste de un estanque de cultivo $(3.0 \mathrm{~m}$ de diámetro $\times 1.1 \mathrm{~m}$ de profundidad, $7 \mathrm{~m}^{3}$ ) conectado a un sistema de recirculación de agua que se purifica, consecutivamente, a través de un filtro biológico, un filtro mecánico de arena de sílice, un filtro de zeolita y un sistema de purificación con luz ultravioleta (fig. 1) (Spotte, 1979; Anónimo, 1986; Huguenin y Colt, 1989; Timmons y Losordo, 1994). Antes de que la descarga del estanque de cultivo entre al filtro biológico, el agua de mar pasa por un filtro mecánico $\left(1.5 \mathrm{~m}^{3}\right)$ dividido en cinco cámaras rellenas con algodón sintético para retener desechos, partículas de comida y heces (Bückle et al., 1990). La cantidad total de agua recirculada fue de $11 \mathrm{~m}^{3}$ e incluyó la de los estanques menores utilizados para otros propósitos de cultivo. El tiempo medio de residencia del agua en el estanque grande fue de $1.23 \mathrm{~h}$ (cantidad de agua del estanque $\mathrm{m}^{3} \mathrm{~h}^{-1} /$ volumen del estanque m³; Anónimo, 1986).

La superficie de pared y el área de fondo $\left(13.9 \mathrm{~m}^{2}\right)$ de la unidad de cultivo de $7 \mathrm{~m}^{3}$ se incrementó a $84.6 \mathrm{~m}^{2}$ usando 31 cortinas verticales de malla de mosquitero sostenidas de un anillo de acrílico detenido por el tubo central (figs. 2a-c, 3). La profundidad del agua y el diámetro interno fueron 0.84 y 2.98 $\mathrm{m}$, respectivamente, y el volumen total que se logró fue $5.85 \mathrm{~m}^{3}$. Como difusor de aire se instaló una manguera de irrigación ( $1.3 \mathrm{~cm}$ de diámetro) alrededor del perímetro interno de

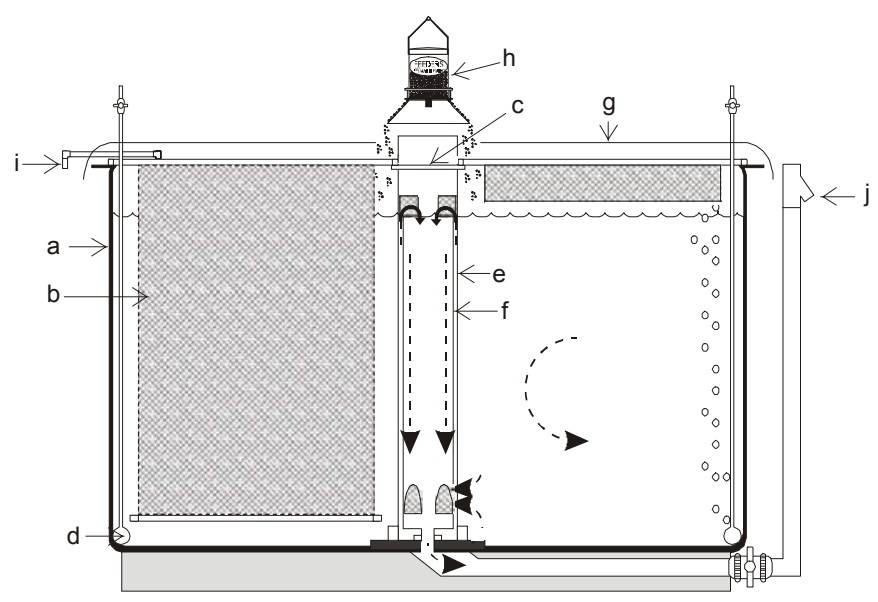

Figure 2. Diagrammatic cross section of the culture tank $\left(7 \mathrm{~m}^{3}\right)$ : (a) black fiberglass high-density culture tank; (b) vertical curtain $(1.0 \times 1.17 \mathrm{~m}) ;(\mathbf{c})$ acrylic ring to uphold the curtains; (d) air diffuser; (e) external PVC pipe (20 $\mathrm{cm} \phi)$ with mesh; (f) internal PVC pipe $(15 \mathrm{~cm} \phi) ;(\mathrm{g})$ plastic sheet; $(\mathbf{h})$ automatic feeder; (i) water supply; (j) outflow. The arrows indicate the seawater flow in the tank.

Figura 2. Sección transversal del estanque de cultivo $\left(7 \mathrm{~m}^{3}\right)$ : (a) estanque de cultivo de fibra de vidrio de alta densidad de color negro; (b) cortina vertical $(1.0 \times 1.17 \mathrm{~m})$; (c) anillo de acrílico para sostener las cortinas; $(\mathbf{d})$ difusor de aire; (e) tubo externo de PVC $(20 \mathrm{~cm} \phi)$ con malla; (f) tubo interno de PVC $(15 \mathrm{~cm} \phi) ;(\mathbf{g})$ cubierta de plástico; (h) alimentador automático; (i) entrada de agua; (j) descarga. Las flechas indican la circulación del agua de mar en el estanque. 


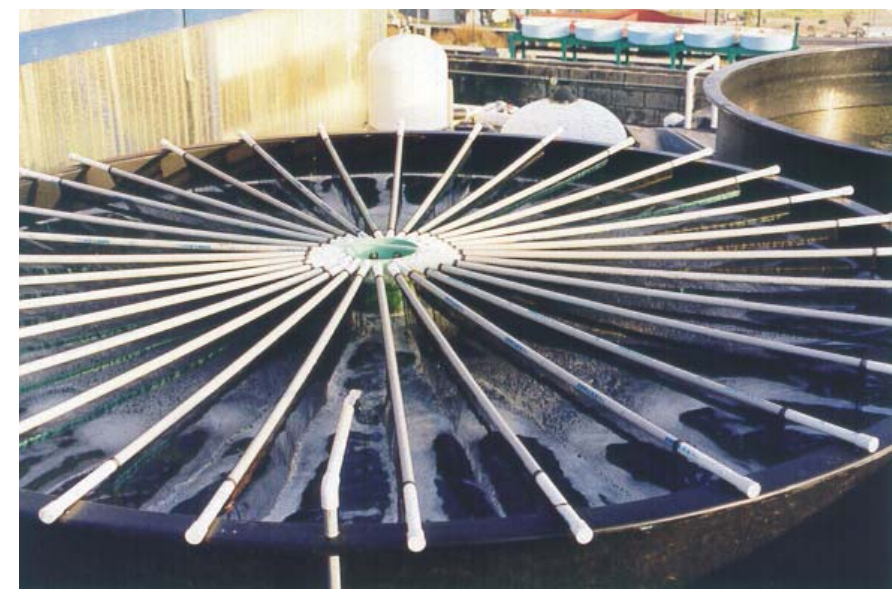

Figure 3. General view of the culture unit $\left(7 \mathrm{~m}^{3}\right)$ for Litopenaeus vannamei. Note the central curtain support.

Figura 3. Vista general del dispositivo de cultivo $\left(7 \mathrm{~m}^{3}\right)$ para Litopenaeus vannamei. Note el apoyo central para las cortinas.

and internal diameter were 0.84 and $2.98 \mathrm{~m}$, respectively, and the total effective volume was $5.85 \mathrm{~m}^{3}$. An irrigator soaker (1.3 $\mathrm{cm}$ diameter) was installed around the internal base perimeter of the tank as an air diffuser to maintain appropriate aeration generated by air blowers (fig. 2d). Additionally, an automatic system with pure oxygen was installed to cope with electricity shortage (fig. 1).

The central PVC pipe $(20 \mathrm{~cm}$ diameter) of the tank had mesh screens at the base and at the water level in order to keep shrimp from escaping (fig. 2e). The internal PVC pipe (15 cm diameter) maintained the water level of the culture tank (fig. 2f). The water recirculation rate was $7.2 \mathrm{~m}^{3} \mathrm{~h}^{-1}$. A transparent plastic sheet was installed over the tank surface to prevent shrimp from jumping out (fig. $2 \mathrm{~g}$ ).

\section{Culture trial of Litopenaeus vannamei}

Shrimp postlarvae (PL 13), with an average individual mass of $4.8 \mathrm{mg}$ (according to the provider), were acquired from the Maricultura del Pacifico aquaculture facility in the state of Sinaloa, Mexico, in the southern part of the Gulf of California $\left(23^{\circ} 13^{\prime} \mathrm{N}, 106^{\circ} 25^{\prime} \mathrm{W}\right)$. They were raised at $20^{\circ} \mathrm{C}$ and $24 \%$.

Immediately after arrival to our laboratory, they were slowly transferred over $2 \mathrm{~h}$ to the culture tank under initial conditions of $24^{\circ} \mathrm{C}$ and $28 \%$. Shrimp (approximately 33,400 PL) were cultivated during 168 days in a $5.85-\mathrm{m}^{3}$ rearing unit. Afterwards, the temperature was increased over five days to $28^{\circ} \mathrm{C}$ using two $1000-\mathrm{W}$ stainless steel heaters regulated with an electronic device. During the first month, shrimp were fed twice a day with finely ground Camaronina (Obregón, Mexico; $35 \%$ protein content) at a rate of $170 \mathrm{~g}$ mixed with $1.0 \mathrm{~L}$ of seawater per day, and then continuing with Camaronina pellets with the same protein content and at the same rate apportioned through an automatic feeder twice a day at 09:00 and 14:00 h (fig. 2h). Different species of algae grew on the light-exposed la base del estanque para mantener una aireación adecuada a través de sopladores (fig. 2d). Además, se instaló un sistema automático de suministro de oxígeno puro en caso de un corte de electricidad (fig. 1).

El tubo de PVC central $(20 \mathrm{~cm}$ de diámetro) del estanque tenía mallas en la base y al nivel del agua para impedir que se escaparan los camarones (fig. 2e). El tubo de PVC interno (15 cm de diámetro) sirvió para mantener el nivel del agua en el estanque de cultivo (fig. 2f). La tasa de recirculación del agua fue de $7.2 \mathrm{~m}^{3} \mathrm{~h}^{-1}$. Se instaló una cubierta de plástico sobre la superficie del estanque para evitar la pérdida de camarones (fig. $2 \mathrm{~g}$ ).

\section{Experimento de cultivo de Litopenaeus vannamei}

Se adquirieron postlarvas (PL 13) de camarón de la empresa Maricultura del Pacífico en el estado de Sinaloa, México, en la parte sur del Golfo de California $\left(23^{\circ} 13^{\prime} \mathrm{N}\right.$, $\left.106^{\circ} 25^{\prime} \mathrm{W}\right)$. Estos organismos, con una masa individual media de $4.8 \mathrm{mg}$ (según el proveedor), fueron criados a $20^{\circ} \mathrm{C}$ y $24 \%$.

Al llegar al laboratorio fueron transferidos de inmediato, aunque poco a poco durante $2 \mathrm{~h}$, al estanque de cultivo, bajo condiciones iniciales de $24^{\circ} \mathrm{C}$ y $28 \%$. Los camarones (aproximadamente 33,400 PL) se cultivaron durante 168 días en una unidad de cultivo de $5.85 \mathrm{~m}^{3}$. Posteriormente, durante cinco días se incrementó la temperatura hasta $28^{\circ} \mathrm{C}$ usando dos calentadores de acero inoxidable de $1000 \mathrm{~W}$ regulados electrónicamente. Durante el primer mes los camarones fueron alimentados dos veces al día con Camaronina molida (Obregón, México; contenido de proteína de 35\%), a una tasa de 170 g mezclada con $1.0 \mathrm{~L}$ de agua de mar por día; después se alimentaron con Camaronina peletizada con el mismo contenido de proteína y a la misma tasa, que se suministró de manera automática en el estanque dos veces al día, a las 09:00 y 14:00 h (fig. 2h). Durante todo el experimento crecieron sobre la malla de mosquitero expuesta a la luz y sobre la pared interna de la unidad de cultivo diferentes especies de algas, las cuales aparentemente fueron utilizadas por los camarones como comida adicional. Se inspeccionó el fondo de la unidad periódicamente con un tubo de PVC de $6.0 \mathrm{~cm}$ cubierto de un lado con acrílico claro, y se tomaron muestras del fondo para determinar la acumulación de desechos. La unidad de cultivo estuvo expuesta a un fotoperiodo natural de primavera y verano.

La salinidad se midió diariamente con un refractómetro (Vista, modelo A366ATC $\pm 1.0 \%$ ) y se ajustó a $28 \%$ agregando agua de mar o agua dulce al sistema de recirculación según fuera necesario. La temperatura y la concentración de oxígeno se registraron con un medidor de oxígeno (YSI, modelo 50B). Al final del experimento (octubre) se determinó la calidad del agua en las diferentes unidades del sistema de la siguiente manera: amoniaco con el método del salicilato, nitrito con el método de diazotización, nitrato con el método de reducción de cadmio, y fosfato y fósforo total con los métodos del molibdovanadato y digestión con persulfato (Anónimo, 1992). 
mosquito screen and on the internal wall of the rearing unit throughout the experiment, and were apparently used by shrimp as supplementary food. The bottom of the rearing unit was periodically inspected with a $6.0 \mathrm{~cm}$ PVC pipe covered at one end with clear acrylic, and bottom samples were also taken in order to examine them for waste accumulation. The culture unit was exposed to natural spring and summer photoperiods.

Salinity was measured with a refractometer (Vista model A366ATC $\pm 1.0 \%$ o on a daily basis and adjusted to $28 \%$ by adding either sea or tap water to the recirculation system. Temperature and oxygen concentration were measured with an oxygen meter (YSI model 50B). At the end of the experiment (October), water quality in the different units that comprised the culture system was measured as follows: ammonia with the salicylate method, nitrite with the diazotization method, nitrate with the cadmium reduction method, and phosphate and total phosphorus with the molybdovanadate and acid persulfate digestion methods (Anonymous, 1992).

Shrimp samples were taken at random from the rearing unit nine times during the experiment. Shrimp length was measured with an electronic caliper (Max-Cal $\pm 0.03 \mathrm{~mm}$ ) from the tip of the rostrum to the tip of the telson, and each organism was weighed in an electronic Ohaus balance $( \pm 0.001 \mathrm{~g})$. Instantaneous apparent feed conversion ratio (AFCR) (Hepher, 1988) was calculated as the apportioned food/mass gained for each sampling period throughout the experiment.

\section{Results}

\section{Culture system operation}

The design of the reservoir for shrimp culture provided good performance. The amount of water flow through the rearing units was excellent in terms of the maintenance and cleanliness of the bottom. Ammonia $\left(\mathrm{NH}_{3}\right)$ in the rearing tank effluent was $0.8 \mathrm{mg} \mathrm{L}^{-1} \mathrm{NH}_{3}-\mathrm{N}$ and, after water passed through the biological filter, it was reduced to $0.3 \mathrm{mg} \mathrm{L} \mathrm{L}^{-1} \mathrm{NH}_{3}-\mathrm{N}$ (63\%) (table 1). Seawater at $28 \%, 28^{\circ} \mathrm{C}$ and $\mathrm{pH} 7.4$ contains $0.012 \mathrm{mg} \mathrm{L}^{-1} \mathrm{NH}_{3}-\mathrm{N}$ (Anonymous, 1986).

Table 1. Water quality of the recycling rearing system for Litopenaeus vannamei culture at the end of the trial. A, seawater; B, biofilter effluent; and $\mathrm{C}$, rearing tank outlet.

Tabla 1. Calidad del agua del sistema de recirculación para el cultivo de Litopenaeus vannamei al final del experimento. A, agua de mar; B, descarga del biofiltro; y $\mathrm{C}$, desagüe del estanque de cultivo.

\begin{tabular}{|c|c|c|c|}
\hline & A & B & $\mathrm{C}$ \\
\hline $\mathrm{pH}$ & 7.200 & 7.300 & 7.400 \\
\hline $\mathrm{NH}_{3}-\mathrm{N} \mathrm{mg} \mathrm{L}^{-1}$ & 0.100 & 0.300 & 0.800 \\
\hline $\mathrm{NO}_{2}-\mathrm{N} \mathrm{mg} \mathrm{L}{ }^{-1}$ & 0.008 & 0.310 & 0.287 \\
\hline $\mathrm{PO}_{4} \mathrm{mg} \mathrm{L}^{-1}$ & - & 7.980 & 7.900 \\
\hline $\mathrm{PO}_{4}$ total $\mathrm{mg} \mathrm{L}^{-1}$ & - & 2.110 & 1.710 \\
\hline
\end{tabular}

Se tomaron muestras de camarones al azar de la unidad de cultivo nueve veces durante el experimento. Los organismos se midieron con un calibrador electrónico (Max-Cal $\pm 0.03 \mathrm{~mm}$ ) de la punta del rostro a la punta del telson y se pesaron en una balanza electrónica (Ohaus $\pm 0.001 \mathrm{~g}$ ). A lo largo del experimento se calculó la tasa instantánea aparente de conversión de alimento (AFCR) (Hepher, 1988) como la proporción de comida/incremento de masa para cada periodo de muestreo.

\section{Resultados}

\section{Operación del sistema de cultivo}

El diseño del tanque para cultivo de camarones funcionó bien. La cantidad de agua recirculada a través de las unidades de cultivo fue excelente en términos del mantenimiento y limpieza del fondo. El contenido de amoniaco $\left(\mathrm{NH}_{3}\right)$ en la descarga del estanque de cultivo fue de $0.8 \mathrm{mg} \mathrm{L}^{-1} \mathrm{NH}_{3}-\mathrm{N}$, reduciéndose a $0.3 \mathrm{mg} \mathrm{L}^{-1} \mathrm{NH}_{3}-\mathrm{N}(63 \%)$ después de pasar el agua por el filtro biológico (tabla 1). El agua de mar a 28\%o, $28^{\circ} \mathrm{C}$ y pH 7.4 contiene $0.012 \mathrm{mg} \mathrm{L}^{-1} \mathrm{NH}_{3}-\mathrm{N}$ (Anónimo, 1986).

La instalación de una unidad de circulación de aire con tuberías sumergidas permitió mantener un contenido alto de oxígeno (5.3-7.5 $\mathrm{mg} \mathrm{L}^{-1}$; tabla 2) y una excelente circulación de agua, impidiendo la acumulación de heces y desechos. Mediante la inspección periódica del fondo del estanque se verificó que no hubo acumulación ni de heces ni de comida.

Durante el experimento la temperatura, aunque estable, no se pudo mantener a $28^{\circ} \mathrm{C}$ como se había propuesto, ya que las temperaturas ambiente durante esa primavera y ese verano fueron menores en comparación con las de otros años, por lo que con los dos calentadores de $1000 \mathrm{~W}$ sólo se pudo mantener la temperatura de $23^{\circ} \mathrm{C}$ a $25.7^{\circ} \mathrm{C}$ (tabla 2). La salinidad se mantuvo al nivel objetivo de $28 \%$ (tabla 2). En septiembre el contenido de oxígeno disminuyó a $0.72 \mathrm{mg} \mathrm{L}^{-1}$ a causa de una falla en el suministro eléctrico, pero no se encontraron camarones muertos; sin embargo, después de este evento se instaló un sistema de oxígeno que lo compensara automáticamente en caso de otra interrupción del suministro.

Table 2. Monthly means ( $\pm S E$ ) temperature, salinity and oxygen content of recirculating seawater in the Litopenaeus vannamei culture.

Tabla 2. Promedios mensuales ( $\pm E E$ ) de temperatura, salinidad y contenido de oxígeno del agua de mar recirculada para cultivar Litopenaeus vannamei.

\begin{tabular}{lccc}
\hline & $\begin{array}{c}\text { Temperature } \\
\left({ }^{\circ} \mathrm{C}\right)\end{array}$ & $\begin{array}{c}\text { Salinity } \\
(\%)\end{array}$ & $\begin{array}{c}\text { Oxygen } \\
\left(\mathrm{mg} \mathrm{L}^{-1}\right)\end{array}$ \\
\hline May & $23.0 \pm 0.4$ & $28.3 \pm 0.2$ & $7.5 \pm 0.08$ \\
June & $25.8 \pm 0.2$ & $28.4 \pm 0.2$ & $6.9 \pm 0.15$ \\
July & $25.3 \pm 0.2$ & $27.5 \pm 0.2$ & $5.8 \pm 0.08$ \\
August & $25.7 \pm 0.2$ & $27.6 \pm 0.2$ & $5.4 \pm 0.09$ \\
September & $24.7 \pm 0.2$ & $28.2 \pm 0.3$ & $5.3 \pm 0.14$ \\
October & $25.4 \pm 0.2$ & $28.3 \pm 0.2$ & $5.9 \pm 0.13$ \\
\hline
\end{tabular}


The installation of an air circulation unit made with water soaker-tubing maintained a high oxygen level (5.3-7.5 $\mathrm{mg} \mathrm{L}^{-1}$; table 2) and excellent water circulation, preventing excreta and waste material from building up. Periodic inspection of the tank bottom revealed that neither faeces nor food had accumulated.

During the experiment, the temperature, although stable, could not be maintained at the planned $28^{\circ} \mathrm{C}$ because the spring-summer ambient temperature was lower compared to other years. As a result, the two $1000-\mathrm{W}$ heaters were only able to maintain $23^{\circ} \mathrm{C}-25.7^{\circ} \mathrm{C}$ (table 2). Salinity of the seawater was maintained at our target level of $28 \%$ (table 2). In September, an electricity shortage caused the oxygen content to drop to $0.72 \mathrm{mg} \mathrm{L}^{-1}$, but we did not find any dead shrimp. After this extreme event, we installed an emergency pure oxygen automatic system to compensate for electrical failure.

\section{Production of Litopenaeus vannamei, apparent food conversion rate, and growth}

The final crop for the rearing unit was $56.7 \mathrm{~kg}$, with a shrimp mean mass of $4.0 \mathrm{~g}$, and a minimum and maximum of 0.4 and $8.5 \mathrm{~g}$, respectively. Dead or predated organisms during 168 days represented $42.5 \%$. In terms of the total recirculating seawater volume, the production was $5.15 \mathrm{~kg} \mathrm{~m}^{-3}$. If only the culture tank volume $\left(5.85 \mathrm{~m}^{3}\right)$ is considered, the production was $9.7 \mathrm{~kg} \mathrm{~m}^{-3}$. In this rearing unit, the initial density of postlarvae was 395 shrimp $\mathrm{m}^{-2}$. Calculated from the initial shrimp mean mass $(4 \mathrm{~g})$ of the crop and the effective surface area with curtains, the final density was 200 shrimp $\mathrm{m}^{-2}$.

Although shrimp mass at the end of the culture had increased (fig. 4), the mean length revealed that the organisms did not grow at the normal rate. The last sample, taken 32 days

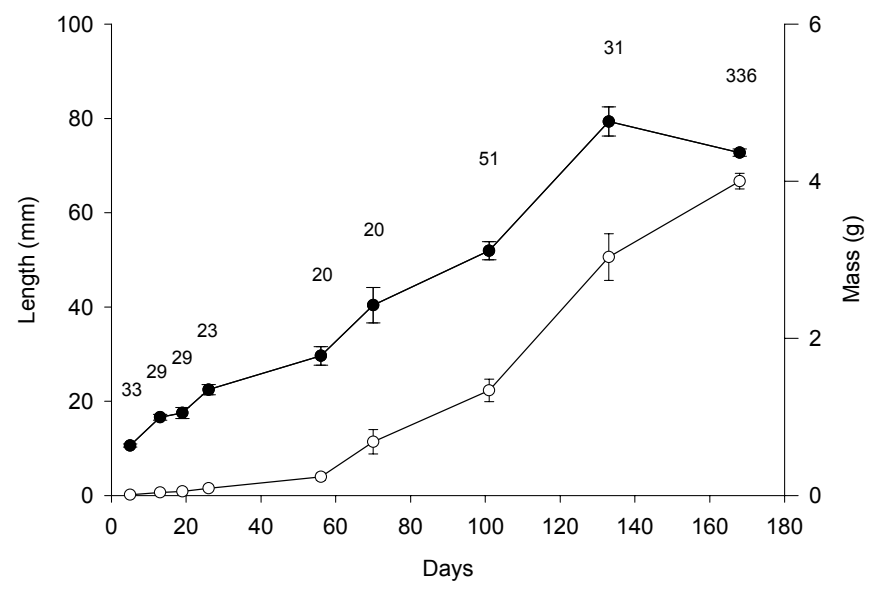

Figure 4. Mean length of Litopenaeus vannamei $(\bullet)$ and mass $(\mathrm{O})( \pm \mathrm{SE})$ increase from May to October 1999 for the $5.85-\mathrm{m}^{3}$ rearing unit. Numbers depict sample size.

Figura 4. Longitud media de Litopenaeus vannamei $(\bullet)$ e incremento de la masa (o) ( $\pm \mathrm{EE}$ ) de mayo hasta octubre de 1999 en la unidad de cultivo de $5.85 \mathrm{~m}^{3}$. Los números indican el tamaño de la muestra.

\section{Producción de Litopenaeus vannamei, tasa aparente de conversión de alimento y crecimiento}

La cosecha final del estanque de cultivo fue de $56.7 \mathrm{~kg}$, con una masa media de los camarones de $4.0 \mathrm{~g}$, y un mínimo y máximo de 0.4 y $8.5 \mathrm{~g}$, respectivamente. Los organismos muertos o depredados durante 168 días correspondieron a $42.5 \%$. En términos del volumen total de agua recirculada, la producción fue de $5.15 \mathrm{~kg} \mathrm{~m}^{-3}$. Si sólo se considera el volumen del estanque $\left(5.85 \mathrm{~m}^{3}\right)$, la producción fue de $9.7 \mathrm{~kg} \mathrm{~m}^{-3}$. En esta unidad de cultivo, la densidad inicial de postlarvas fue de 395 camarones $\mathrm{m}^{-2}$. La densidad final, calculada a partir de la masa inicial media de los camarones ( $4 \mathrm{~g})$, la cosecha y el área de superficie efectiva con cortinas, fue de 200 camarones $\mathrm{m}^{-2}$.

Aunque la masa de los camarones al final del cultivo había amentado (fig. 4), la longitud media de los organismos reveló que éstos no crecieron a la tasa normal. La última muestra, tomada 32 días después de la caída abrupta de oxígeno, revela que este evento ocasionó estrés en los camarones, ya que muestras anteriores habían indicado que el cultivo procedía favorablemente. Además, consideramos que la cantidad de alimento proporcionada fue insuficiente dado que en la última muestra se encontró que el AFCR había aumentado de 1.5 a 23.4 (tabla 3). La cantidad total de alimento proporcionado al cultivo fue de $130.9 \mathrm{~kg}$ y la AFCR fue de 2.3. Parte de la comida se perdió debido a que el filtro mecánico siempre contenía partículas de alimento. Sobre las paredes del estanque se desarrollaron cinco especies de macroalgas que representaron una fuente de alimento adicional para los camarones. Los camarones fueron observados aparentemente raspando las algas que cubrían las cortinas, pero no se llevó a cabo un análisis del consumo de estas algas. No se detectaron organismos infectados, por lo que el crecimiento no fue afectado por enfermedad alguna.

Table 3. Instant apparent food conversion rate (AFCR) of Litopenaeus vannamei cultivated in a $5.85-\mathrm{m}^{3}$ rearing unit for 168 days.

Tabla 3. Tasa instantánea aparente de la conversión de alimento (AFCR) de Litopenaeus vannamei cultivado en una unidad de $5.85 \mathrm{~m}^{3}$ por 168 días.

\begin{tabular}{lc}
\hline Sample & $\begin{array}{c}\text { Instant AFCR } \\
\text { (food/mass) }\end{array}$ \\
\hline 25-31 May & 7.0 \\
1-7 June & 2.0 \\
8-14 June & 7.3 \\
15-21 June & 1.6 \\
22-5 July & 2.9 \\
6-19 July & 1.3 \\
20-12 August & 2.0 \\
13-20 September & 1.5 \\
21-25 October & 23.4 \\
\hline
\end{tabular}


after the extreme oxygen drop, indicated that this event produced stress in the shrimp culture, particularly because previous samples indicated that the culture was growing well. In addition, we consider that the food quantity apportioned to the culture was insufficient because the last sample showed that the instantaneous AFCR increased from 1.5 to 23.4 (table 3). The total amount of food apportioned to the culture was $130.9 \mathrm{~kg}$ and the AFCR was 2.3. Part of the food was lost because the mechanical filter always contained uneaten food particles. Five species of macroalgae that the shrimp could consume as a supplemental food source developed on the walls of the rearing unit; we observed shrimp apparently scraping the algae covering the curtain surface, but we did not test for algal consumption. Growth was no affected by disease, since we did not detect infected organisms.

\section{Discussion}

The design and installation of mesh curtains is a viable tank modification to increase available space in a reduced water volume. The mesh provides a large surface where shrimp can walk or rest. Shrimp on both surfaces of a curtain were well distributed and for that reason we consider that stress in the culture was reduced.

Zelaya et al. (2001) described a similar culture system utilizing one $L$. vannamei production pond with water recirculated through a treatment pond. This is the same basic system as in the present trial. The treatment pond corresponds to the biofilter where water is filtered and purified before returning to the culture tank. To avoid pathogenic disease invasion, Shiau $e t$ al. (2001) conducted outdoor pond culture using integrated water treatments comprising a sedimentation area, a submerged biofilter, and a pump base with airlift. In our 168-day trial, we did not have any infection, probably due to complete control of water quality and the small amount of water refill to the culture.

Velasco et al. (2001) emphasized water management and nutrition strategy for postlarvae reared in static and recirculating culture systems. Our results indicate that a 1.23 -h mean residence time of the recirculated water in the rearing unit can sustain high water quality. During the final stage of the trial, concentrations of total and un-ionized ammonia ( 0.8 and $0.012 \mathrm{mg} \mathrm{L}^{-1}$, respectively) were lower than the lethal concentrations (LC50) of 1.69, 1.2 and $1.1 \mathrm{mg} \mathrm{NH}_{3}-\mathrm{N} \mathrm{L}^{-1}$ measured for Penaeus monodon (Allan et al., 1990), P. paulensis PérezFarfante 1967 (Ostrensky and Wasielesky, 1995), and $P$. setiferus Linnaeus 1767 (Alcaraz et al., 1999), respectively. In the present study, L. vannamei grew in a very low ammonia concentration environment compared with the concentrations of 0.21 and $1.39 \mathrm{mg} \mathrm{NH}_{3}-\mathrm{N} \mathrm{L}^{-1}$ that reduced the growth of $P$. monodon and P. japonicus Bate 1888 to 5\% and 50\%, respectively (Allan et al., 1990; Chen and Kou, 1992).

Troell et al. (1999) co-cultivated Gracilaria with salmon where macroalgae could remove $50 \%$ and $90-95 \%$ of the dissolved ammonium released in winter and spring, respectively.

\section{Discusión}

El diseño y la instalación de las cortinas de malla es una modificación viable para incrementar el espacio disponible en un volumen de agua reducido. La malla ofrece una superficie grande sobre la cual pueden caminar o descansar los camarones. Los camarones estuvieron bien distribuidos en ambas superficies de la cortina y por tal razón se considera que fue poco el estrés al que estuvieron sometidos.

Zelaya et al. (2001) describieron un sistema de cultivo similar utilizando un estanque de producción de L. vannamei con agua recirculada a través de un estanque de tratamiento. Este sistema es básicamente igual al usado en este experimento ya que el estanque de tratamiento corresponde al biofiltro donde el agua es filtrada y purificada antes de regresar al estanque de cultivo. Para evitar la invasión de enfermedades patogénicas, Shiau et al. (2001) realizaron cultivos en estanques al aire libre usando tratamientos integrados de agua que comprenden un área de sedimentación, un biofiltro sumergido y una bomba de aire comprimido. En nuestro experimento de 168 días no se presentaron infecciones, probablemente debido al control total de la calidad del agua y al poco recambio de ésta.

Velasco et al. (2001) mencionaron la importancia del manejo del agua y la estrategia de nutrición en el cultivo de postlarvas en sistemas de recirculación y estáticos. Nuestros resultados muestran que un tiempo de residencia medio de $1.23 \mathrm{~h}$ del agua recirculada en la unidad de cultivo puede mantener una alta calidad del agua. En la etapa final del experimento las concentraciones de amonio total y no ionizado ( 0.8 y $0.012 \mathrm{mg} \mathrm{L}^{-1}$, respectivamente) fueron menores que las concentraciones letales (LC50) de 1.69, 1.2 y $1.1 \mathrm{mg} \mathrm{NH}_{3}-\mathrm{N} \mathrm{L}^{-1}$ registradas para Penaeus monodon (Allan et al., 1990), P. paulensis Pérez-Farfante 1967 (Ostrensky y Wasielesky, 1995) y P. setiferus Linnaeus 1767 (Alcaraz et al., 1999), respectivamente. En el presente estudio la concentración de amoniaco en el ambiente en que creció $L$. vannamei fue muy baja comparada con las concentraciones de 0.21 y $1.39 \mathrm{mg} \mathrm{NH}_{3}-\mathrm{N} \mathrm{L}^{-1}$ que redujeron el crecimiento de $P$. monodon y $P$. japonicus Bate 1888 a $5 \%$ y $50 \%$, respectivamente (Allan et al., 1990; Chen y Kou, 1992).

En el cocultivo de Gracilaria con salmón realizado por Troell et al. (1999), las macroalgas eliminaron 50\% y $90-95 \%$ del amonio disuelto liberado en invierno y primavera, respectivamente. Asimismo, se han utilizado pastos marinos en estanques de cultivo de $P$. chinensis Osbeck 1765 para mejorar la calidad del agua y la producción de camarón (Ren et al., 1991).

Porchas et al. (1995) estudiaron la relación entre la supervivencia de juveniles de P. californiensis Holmes 1900 cultivados en estanques y el crecimiento de Caulerpa sertularioides. La masa y la supervivencia de los camarones se incrementaron en presencia del alga por encima de las observadas sin ella. Estos autores sugirieron una posible asociación 
Furthermore, eelgrasses have been added to $P$. chinensis Osbeck 1765 culture ponds to improve water quality and shrimp production (Ren et al., 1991).

Porchas et al. (1995) studied the relation between survival of tank-cultured juvenile $P$. californiensis (Holmes, 1900) and the growth of live Caulerpa sertularioides. Mass and survival of shrimp increased with the presence of algae over that observed without algae. Porchas et al. (1995) proposed that a biological association could exist between the organisms and macroalgal growth. In this trial, we believe that the growth of algae in the rearing tanks helped to maintain water quality at a safe level and was probably used as an additional food source. We found various species of macroalgae, including Enteromorpha compressa (Linnaeus) Greville, E. linza (Linnaeus) J. Agardh, Ulva lactuca Linnaeus, Cladophora sakai Abbott, and Oscillatoria sp., that are eaten by $P$. japonicus (Reymond and Lagardère, 1990). Our curtains have a similar effect to that observed for Aqua-Mats ${ }^{\mathrm{TM}}$ (Marin et al., 1999) in supporting natural feed for postlarvae and juveniles.

Tacon et al. (2002) contrasted eight-week feeding trials with juvenile shrimp cultivated indoors to outdoor "green water" culture conditions. They observed higher growth and feed performance outdoors, probably caused by the food produced endogenously. Bradvolt and Browdy (2001) stocked tanks with postlarval shrimp, sand and hanging mats as additional vertical surface. Their experiments suggested benefits from natural production during early growth stages. In this trial, we consider that the natural tank feed production was directly integrated as part of the shrimp-feeding regime. Therefore, the calculated food conversion efficiency for this test is only an estimate (table 3 ).

Each successive sample removed from the rearing unit indicated that the mass of the culture was doubling and the shrimp growth trend was adequate, up to the penultimate sample where the AFCR increased apparently because of stress caused by food shortage and anoxia. The AFCR (2.41) is high compared with that observed by Aragón and Calderón (1997) using earthen pond intensive culture of white shrimp stocked at 92-109 $\mathrm{m}^{-2}$ (AFCR = 1.11). Casillas and Villarreal $(1995 \mathrm{~b})$ obtained a mean food conversion ratio of 1.8-2.0 for a threeyear study in a 44 ha experimental farm. Paquotte et al. (1998) obtained a food conversion efficiency average of 2.58-3.15 using growing cages installed in the estuarine zone. None of these authors discusses probable growth enhancement by macroalgae or biofouling in the cages.

Literature concerning recycling systems for the culture of L. vannamei is scarce, and the systems and conditions of culturing are very diversified. It is only possible to make a comparison taking into account production per $\mathrm{kg} \mathrm{m}^{-2}$ crop $^{-1}$. In this sense, shrimp production has improved from around 1 to $7-8 \mathrm{~kg} \mathrm{~m}^{-2}$ crop $^{-1}$ in the last few years (table 4 ).

In this experiment, shrimp production was $8.1 \mathrm{~kg} \mathrm{~m}^{-2}$ of base area (excluding mesh curtains). This production was very biológica entre los organismos y el crecimiento de la macroalga. En nuestro experimento, la presencia de algas en los tanques de cultivo aparentemente ayudó a mantener la calidad del agua en un nivel seguro, además de que probablemente fueron utilizadas como fuente de alimento adicional. Encontramos varias especies de macroalgas, incluyendo Enteromorpha compressa (Linnaeus) Greville, E. linza (Linnaeus) J. Agardh, Ulva lactuca Linnaeus, Cladophora sakai Abbott, y Oscillatoria sp., las cuales son consumidas por P. japonicus (Raymond y Lagardère, 1990). Las cortinas utilizadas en este estudio tienen un efecto similar al observado con Aqua-Mats ${ }^{\mathrm{TM}}$ (Marin et al., 1999) en el sustento de alimento natural para postlarvas y juveniles.

Tacon et al. (2002) contrastaron ensayos de alimentación de camarones juveniles de ocho semanas bajo techo con cultivos en "agua verde" al aire libre. Observaron mayor crecimiento y rendimiento alimenticio al aire libre, probablemente debido a la producción endógena del alimento. Los experimentos de Bradvolt y Browdy (2001), en un sistema de cultivo de postlarvas de camarón con fondo de arena y cortinas verticales como superficie adicional, mostraron los beneficios de la producción natural durante las etapas tempranas de crecimiento. En el presente estudio se considera que la producción de alimento natural en el estanque estuvo directamente integrada al régimen de nutrición; por tanto, la eficiencia de conversión de alimento calculada para este experimento es tan sólo una aproximación (tabla 3 ).

Cada muestra sucesiva tomada del estanque de cultivo indicó que la masa del cultivo se duplicaba y que el crecimiento de los camarones era adecuado, hasta la penúltima muestra, cuando la AFCR aumentó aparentemente a causa del estrés producido por la escasez de alimento y la anoxia. La AFCR (2.41) es alta en comparación con la observada por Aragón y Calderón (1997) en el cultivo intenso de camarón blanco en estanques de tierra sembrados a una densidad de 92 a $109 \mathrm{~m}^{-2}($ AFCR $=1.11)$. En un estudio de tres años en una granja experimental de 44 ha, Casillas y Villarreal (1995b) obtuvieron una tasa de conversión de alimento de 1.8 a 2.0 en promedio, mientras que Paquotte et al. (1998) obtuvieron una eficiencia de conversión de alimento media de 2.58 a 3.15 usando jaulas flotantes instaladas en la zona estuarina. Ninguno de estos autores discute la posibilidad de mayor crecimiento debido a macroalgas o biocontaminación en las jaulas.

Existe poca literatura sobre sistemas de recirculación para el cultivo de L. vannamei, y los sistemas y las condiciones de cultivo son muy diversos. Sólo es posible hacer comparaciones si se toma en cuenta la producción por $\mathrm{kg} \mathrm{m}^{-2}$ cosecha $^{-1}$. En este sentido, la producción de camarón se ha incrementado de alrededor de 1 a $7-8 \mathrm{~kg} \mathrm{~m}^{-2} \operatorname{cosecha}^{-1}$ en los últimos años (tabla 4).

En el presente estudio, la producción de camarón fue de $8.1 \mathrm{~kg} \mathrm{~m}^{-2}$ de área base (excluyendo las cortinas). Esta producción es muy similar a la obtenida por Davis y Arnold 
Barón et al.: Intensive culture of Litopenaeus vannamei in recirculating seawater

Table 4. Production of Litopenaeus vannamei in different recirculating systems. PL = postlarvae $\mathrm{m}^{-2}$.

Tabla 4. Producción de Litopenaeus vannamei en diferentes sistemas de cultivo de recirculación de agua. $\mathrm{PL}=$ postlarvas $\mathrm{m}^{-2}$.

\begin{tabular}{|c|c|c|c|}
\hline Initial density & Culture (days) & Production & Author \\
\hline $223-229 \mathrm{PL}$ & 49 & $1.36-1.56 \mathrm{~kg} \mathrm{~m}^{-2} \mathrm{crop}^{-1}$ & Robertson et al. (1992) \\
\hline $970 \mathrm{PL} \mathrm{m}^{-3}$ & 161 & $0.34 \mathrm{~kg} \mathrm{~m}^{-2} \mathrm{crop}^{-1}$ & Reid and Arnold (1992) \\
\hline $2132 \mathrm{PL} \mathrm{m}^{-3}$ & 133 & $0.32 \mathrm{~kg} \mathrm{~m}^{-2} \mathrm{crop}^{-1}$ & \\
\hline $60 \mathrm{PL}$ & 165 & $0.72 \mathrm{~kg} \mathrm{~m}^{-2} \mathrm{crop}^{-1}$ & Sandifer et al. (1993) \\
\hline $\begin{array}{l}\text { 33-49-66 (0.4 g) } \\
\text { shrimp m }{ }^{-2}\end{array}$ & 102 & $1 \mathrm{~kg} \mathrm{cage}^{-1}$ & Casillas and Villarreal (1995b) \\
\hline 92-109 PL & 101 & $1.22-1.43 \mathrm{~kg} \mathrm{~m}^{-2}$ crop $^{-1}$ & Aragón and Calderón (1997) \\
\hline $173-2379 \mathrm{PL}$ & $77-172$ & $4.86-7.9 \mathrm{~kg} \mathrm{~m}^{-2}$ crop $^{-1}$ & Davis and Arnold (1998) \\
\hline $\begin{array}{c}\text { 41-227 }(0.5 \mathrm{~g}) \\
\text { shrimp } \mathrm{m}^{-2}\end{array}$ & $\begin{array}{c}76-153 \\
\text { hot and cool season }\end{array}$ & $0.8 \mathrm{~kg} \mathrm{~m}^{-2}$ cycle $^{-1}$ & Paquotte et al. (1998) \\
\hline $395 \mathrm{PL}$ & 168 & $8.1 \mathrm{~kg} \mathrm{~m}^{-2} \mathrm{crop}^{-1}$ & This work \\
\hline
\end{tabular}

similar to that obtained by Davis and Arnold (1998), although these authors worked with a raceway system of $33.5 \mathrm{~m}^{2}$.

Another extension of our results is to make use of the system design to culture postlarvae to juveniles less than $4 \mathrm{~g}$ for subsequent transfer to culture facilities. However, more research is needed to assess the culture of shrimp and other crustaceans in recirculating systems with our modifications to ascertain the most inexpensive and secure production.

The water recirculation systems for intensive shrimp cultivation have several advantages compared with the open systems. One can have total control of production, of water quality and temperature, of illnesses, and in providing the best environment for the cultured species. Another improvement in this system is the natural food growth in the tank and the huge space increase with the installation of vertical curtains.

However, it is much more important to consider potential reduction in the use of agricultural land that in some countries, such as Mexico, has been transformed into culture ponds in order to increase shrimp production.

\section{Acknowledgements}

This work was supported by the Mexican Federal Government, through regular funding of the Centro de Investigación Científica y de Educación Superior de Ensenada, and the Consejo Nacional de Ciencia y Tecnología, grant 4050-B. We extend our thanks to Raúl Aguilar-Rosas and Luis E. AguilarRosas for the scientific names of the macroalgae.

\section{References}

Alcaraz, G., Chiapa, C.X., Espinoza, V. and Vanegas, C. (1999). Acute toxicity of ammonia and nitrite to white shrimp Penaeus setiferus postlarvae. J. World Aquacult. Soc., 30: 90-96.

Allan, G.L., Maguire, G.B. and Hopkins, S.J. (1990). Acute and chronic toxicity of ammonia to juvenile Matapenaeus macleayi
(1998), aunque estos autores trabajaron con un sistema tipo "raceway" de $33.5 \mathrm{~m}^{2}$.

Otra aplicación de nuestros resultados es utilizar el diseño del sistema para el cultivo de postlarvas a juveniles de menos de $4 \mathrm{~g}$, para transferirlos después a estanques de cultivo. Sin embargo, para determinar la forma de obtener una producción más segura y económica se requiere de mayor investigación para evaluar el cultivo de camarón y otros crustáceos en sistemas de recirculación con las modificaciones propuestas.

Los sistemas de recirculación de agua para el cultivo intensivo de camarón tienen varias ventajas sobre los sistemas abiertos, ya que se pueden controlar la producción, la calidad del agua, la temperatura y las enfermedades, así como proveer el mejor ambiente para las especies cultivadas. Otra ventaja es el crecimiento de alimento natural en el estanque y el considerable aumento de espacio con la instalación de las cortinas verticales.

Sin embargo, es mucho más importante considerar la reducción potencial en la superficie de tierras agrícolas que en algunos países, como México, han sido transformadas en estanques de cultivo para incrementar la producción de camarón.

\section{Agradecimientos}

Este trabajo fue apoyado por el Gobierno Federal de México, a través del financiamiento del Centro de Investigación Científica y de Educación Superior de Ensenada, y el Consejo Nacional de Ciencia y Tecnología, mediante el proyecto 4050-B. Extendemos nuestro agradecimiento a Raúl Aguilar-Rosas y Luis E. Aguilar-Rosas por los nombres científicos de las macroalgas.

Traducido al español por Christine Harris. 
and Penaeus monodon and the influence of low dissolved-oxygen levels. Aquaculture, '91: 265-280.

Anonymous (1986). Flow-through and recirculation systems. Food and Agriculture Organization of the United Nations. Report of the working group on terminology, format and units of measurement. FAO EIFAC Tech. Pap., 49: 1-100.

Anonymous (1992). Hatch Water Analysis Handbook. Loveland, Colorado, $831 \mathrm{pp}$.

Anonymous (1996). List of animal species used in aquaculture. FAO Fish. Circ. No. 914, FIRI/C914.

Anonymous (1997). Review of the state of world aquaculture. FAO Fish. Circ. No. 886, FIRI/C886 (Rev. 1)

Aragón, N.E.A. and Calderón, A.L.E. (1997). Feasibility of intensive shrimp culture in Sinaloa, Mexico. World Aquacult. '97: 64.

Bradvolt, D. and Browdy, C.L. (2001). Effects on sand sediment and vertical surfaces (AquaMats ${ }^{\mathrm{TM}}$ ) on production, water quality, and microbial ecology in an intensive Litopenaeus vannamei culture system. Aquaculture, 195: 81-94.

Bückle, R.L.F., Morales, G.E., Valenzuela, B.F. y Flores, A.N. (1990). Guía general del laboratorio de Acuicultura. Centro de Investigación Científica y Educación Superior de Ensenada (Mexico). Informe Especial CAACNC9001.

Casillas, R. and Villarreal, H. (1995a). Effect of density on the cage culture of the white shrimp Penaeus vannamei in Bahía Guásimas, Sonora, Mexico. Aquaculture, '95, San Diego, California: 236-237.

Casillas, R. and Villarreal, H. (1995b). Semi-intensive culture of the white shrimp Penaeus vannamei in the northwest of Mexico: South coast of Sonora. Aquaculture, '95, San Diego, California: 235.

Chen, J.C. and Kou, Y.Z. (1992). Effects of ammonia on growth and molting of Pennaeus japonicus juveniles. Aquaculture, 104: 249-260.

Davis, D.A. and Arnold, C.R. (1998). The design, management and production of a recirculating raceway system for the production of marine shrimp. Aquacult. Eng., 17: 193-211.

Hepher, B. (1988). Nutrición de Peces Comerciales en Estanque. Limusa, México, 406 pp.

Huguenin, J.E. and Colt, J. (1989). Design and Operating Guide for Aquaculture Seawater Systems. Elsevier Interscience, Amsterdam, 264 pp.

Marin, R.E., Buitrago, E. y Cabrera, T. (1999). Generación de alimento natural en piscinas de precría del camarón blanco Litopenaeus vannamei mediante el uso de los AquaMats ${ }^{\mathrm{TM}}$. Acuicultura 99, T. Cabrera, D. Jory y M. Silva (eds.), 1: 328-333.

Ogle, T.J. (1991). Design and operation of small tank system for ovarian maturation and spawning of Penaeus vannamei. Gulf Res. Rep., 8: 285-289.

Ostrensky, A. and Wasielesky, W. Jr. (1995). Acute toxicity of ammonia to various life stages of the São Paulo shrimp, Penaeus paulensis Pérez-Farfante, 1967. Aquaculture, 132: 339-347.

Paquotte, P., Chim, L., Martin, J.L.M., Lemos, E., Stern, M. and Tosta, G. (1998). Intensive culture of shrimp Penaeus vannamei in floating cages: Zootechnical, economic and environmental aspects. Aquaculture, 164: 151-166.

Porchas, A.M., Magallon, F., Portillo, G., Naranjo, J., Campos, R. and Villarreal, H. (1995). Direct and indirect effect of the macroalgae Caulerpa sertularioides in the growth and survival of brown shrimp Penaeus californiensis reared at reduced temperatures. Aquaculture '95, San Diego, California: 234-235.

Reid, B. and Arnold, C.R. (1992). The intensive culture of the penaeid shrimp Penaeus vannamei Boone in a recirculating raceway system. J. World Aquacult. Soc., 23: 146-153.

Ren, G., Wang, J., Zhang, Q., and Wang, D. (1991). Transplant eelgrasses in shrimp ponds to increase products of Penaeus chinensis O’sbeck. Mar. Sci./Haiyang Kexue, 1: 52-57.

Reymond, H. and Lagardère, J.P. (1990). Feeding rhythms and food of Penaeus japonicus Bate (Crustacea: Penaeidae) in salt marsh ponds: Role of halophilic entomofauna. Aquaculture, 84: 125-143.

Ricque, D., Martinez, V.J.A. and Aguirre, G.G. (1993). A low cost recirculation synthetic seawater system for nutritional assays in penaeid shrimp. Spec. Publ. Eur. Aquacult. Soc., 19: 161.

Robertson, L., Samocha, T., Gregg, K. y Lawrence, A. (1992). Potencial de engorda postcriadero de Penaeus vannamei en un sistema intensivo tipo "raceway". Cienc. Mar., 18(4): 47-56.

Sandifer, A.P., Hokins, S.J., Stokes, A.D. and Browdy, C.L. (1993). Preliminary comparisons of the native Penaeus setiferus and Pacific $P$. vannamei white shrimp for pond culture in South Carolina, USA. J. World Aquacult. Soc., 24: 295-303.

Shiau, L.J., Chen, I.M. and Chang, M.C. (2001). Design an outdoor recirculating system for the culture of white shrimp Litopenaeus vannamei broodstock. 6th Asian Fisheries Forum, Philippines. Asian Fisheries Society, 304 pp.

Spotte, S. (1979). Fish and Invertebrate Culture. Water Management in Closed Systems. John Wiley, New York, 179 pp.

Tacon, A.G.J., Cody, J.J., Conquest, L.D., Divakaran, S., Forster, I.P. and Decamp, O.E. (2002). Effect of culture system on the nutrition and growth performance of the Pacific white shrimp Litopenaeus vannamei (Boone) fed different diets. Aquacult. Nutr., 8: 121-137.

Timmons, M.B. and Losordo, T.M. (1994). Development in Aquaculture and Fisheries Science, 27. Aquaculture water reuse systems: Engineering design and management. Elsevier, New York, $333 \mathrm{pp}$.

Tirado, M.C., Lotz, J.M., Ogle, J.T. and Youngs, D.W. (1993). Effect of temperature on the growth and survival of Penaeus vannamei postlarvae in closed systems. Spec. Publ. Eur. Aquacult. Soc., 19: 176.

Tirado, M.C., Youngs, W.D., Lotz, J.M. and Ogle, J.T. (1996). Reproduction of the marine shrimp Penaeus vannamei in closed systems. Proc. PACON Conference on Sustainable Aquaculture 95, p. 383.

Troell, M., Roennbaeck, P., Halling, C., Kautsky, N. and Buschman, A. (1999). Ecological engineering in aquaculture: Use of seaweeds for removing nutrients from intensive mariculture. J. Appl. Phycol., 11: 89-97.

Velasco, M., Lawrence, A. and Neill, W.H. (2001). Comparison of survival and growth of Litopenaeus vannamei (Crustacea: Decapoda) postlarvae reared in static and recirculating culture systems. Texas J. Sci., 53: 227-238.

Zelaya, O., Boyd, C.E., Teichert-Coddington, D.R. and Rouse, D.B. (2001). Effects of water recirculation on water quality and bottom soil in shrimp ponds. Aquaculture 2001. World Aquaculture Society, $711 \mathrm{pp}$. 